\title{
ADMISSION CONTROL IN A PURE LOSS HEALTHCARE NETWORK: MDP and DES APPROACH
}

\author{
Canan Pehlivan \\ Vincent Augusto \\ Xiaolan Xie \\ École Nationale Supérieure des Mines de Saint Etienne \\ Center for Health Engineering \\ 158 Cours Fauriel \\ Saint Etienne, 42023, FRANCE
}

\begin{abstract}
This paper considers admission control policies in a pure-loss hierarchical perinatal network where there are 2 parallel multi-server (target) hospitals fed by new arriving patients and overflowed patients from a set of parallel multi-server hospitals. In this perinatal network setting, we consider the problem of finding an optimal admission policy that recommends how many beds to reserve in two target hospitals for each arriving stream in order to maximize total revenue in the system. At first, we assumed a Markovian system and model the system as a Markov Decision Process (MDP). By using value iteration algorithm, optimal admission policy is computed. Afterwards, we evaluate various policy scenarios (including MDP optimal policy) with a simulation model which strengthens the decision making process by incorporating the complexity which can not be captured by MDP and we assess the impact of Markovian assumption in a complex healthcare setting.
\end{abstract}

\section{INTRODUCTION}

The system of perinatal network is a pure loss system. Patients of perinatal networks are urgent, they should not be allowed to wait. Therefore, inadequate capacity in a hospital results in the patient being deferred to another facility which may cause long travel distances to a non-preferred hospital. In other words inadequate capacity in a unit results in rejection and overflow of the patient which might cause fatal occurrences so that rejection probability is considered to be an important performance measure.

Minimization of rejections in such systems has been the subject of many research where commonly scheduling of resources, flow of patients, capacity planning, resource allocation models are studied. In Pehlivan et al. (2012) capacity planning in a perinatal network is addressed and the results demonstrate that capacity and location planning of hospitals leads to serious improvement in the performance of the system whereas those are incredibly costly solutions that are mostly not possible to realize. This reality encouraged us to find a way to improve the system performance in the existing capacity and structure, which can be achieved by controlling the way of system works with some control policies in order to ultimately control its performance.

In a healthcare network, patients themselves choose where to be served and mostly they have their preferred hospitals due to proximity, trustworthy, etc. Furthermore, primary objective of each hospital is to provide service to their own patients. Under these circumstances, the admission of a patient to her first preferred hospital is crucial and considered more profitable than the admission of an overflow patient. As long as the network has sufficient total capacity to meet all demand arose in the region, we can prioritize new arrivals to preferred hospitals over overflow ones, since it is sure all demand will be served. From here, we define new arrivals to the preferred hospitals as class-1, overflow arrivals as class-2 patients. 


\section{Pehlivan, Augusto, and Xie}

Therefore, the objective of this work is to find an optimal admission policy for each class of patients in order to maximize the total reward in the network while not deteriorating the total rejection in the network.

This work is studied on Perinatal Network Haute-de-Seine. In the next section, the contribution aimed with this work is described along with a literature review. In Section 3, the considered perinatal network is characterized along with necessary assumptions and case study parameters. In Section 4, an MDP model is constructed. Admission control value function is given, and the optimal policy is found computationally by using the value iteration algorithm. In Section 5, by using simulation model, several scenarios are generated around the base scenario and evaluated. And finally, conclusion is given.

\section{LITERATURE REVIEW}

Healthcare networks are special structures where complexity is quite high due to the fact that "people serve people" meaning people are both the customer and the supply (Roberts 2011). In such a complex organization, simulation has found widespread application in healthcare literature and is also used as a convenient tool in many studies on healthcare network. Charfeddine and Montreuil (2010) introduced a global framework for integrated agent-oriented modeling through the Chronic Obstructive Pulmonary Disease (COPD) population and healthcare delivery network in Quebec. Brailsford (2007) proposed a classification of discrete-event simulation and systems dynamics in healthcare, based on the level of detail on which the model focuses. Miller, Ferrin, and Shahi (2009) utilized simulation to determine the impact of various patient surge levels on three regional Emergency Departments. Their simulation model pointed out that handling surge depends largely on the percentage of available inpatient beds that a hospital staffs and model also identified the occupancy levels when a hospital should increase in-patient beds.

On the other side MDP models are strong descriptive models that are able to evaluate and predict the performance of existing and proposed systems, and thus are able to improve the design of a system, however requires quite a number of assumptions. There is a vast literature on using Markov decision processes (MDPs) to analyze control policies in a network structure. Many of the related studies are on communication network field. In literature, control of admissions to network of queues has been widely studied however we have encountered few communication papers that have considered the admission control of arrivals to loss queues. Ku and Jordan (2002) studied admission control in a multiserver loss queue, where the target queue faces a choice of how many servers to reserve for each arriving stream. They seek the control policy on only one target hospital. Delasay, Kolfal, and Ingolfsson (2012) focused on optimal routing in a Markovian finite-source, multi-server queueing system with heterogeneous servers, each with a separate queue. They formulate the problem of routing as a Markov Decision Process, they demonstrated that the Shortest Queue policy is optimal when the servers are homogenenous. In our problem, there are two target hospitals (with new patients and overflow patients arriving from other hospitals) overflow to each other when one of them gets full. In such a system, we seek the optimal admission control policies in both hospitals that suggest how many beds to reserve in which hospital.

The problem of finding an optimal admission policy in such a pure loss healthcare network setting is quite interesting, and to our knowledge it has not been studied in the MDP literature. However, MDP models require quite a number of assumptions, therefore it may not be realistic to use MDP results directly on a healthcare problem due to its high complexity. Supporting and improving the decision making process with a simulation model which can better capture the complexity of the healthcare system is the objective of this study.

\section{PERINATAL NETWORK HAUTS-DE-SEINE}

This case study is realized in perinatal network of Hauts-de-Seine in Ile-de-France where there are 6 maternity facilities; 2 big-sized $(H 1, H 2)$ and 4 small-sized $(H 3, H 4, H 5, H 6)$. In perinatal network each maternity facility is composed of at most three types of service units $s \in\{1,2,3\}$; obstetrics units $(s=1)$ that provide labor services to pregnant women, basic neonatal units $(s=2)$ that provide basic care and neonatal 
intensive care units NICUs $(s=3)$ that provide special care for newborn babies. Inherently, these units interact with each other as women in labor who need obstetrics unit may subsequently require neonatal or NICU services (this relation is utilized for computing arrival rates of neonatal services). Maternity facilities differ according to the type of service unit included and patient overflow may occur only between the same type of service units.

The perinatal network considered in this study is represented in Fig.1 Small-sized hospitals serve only to their own (new) arrivals (class- 1 ). Rejected patients overflow to one of the target hospitals ( $H 1$ and $H 2$ ) where they are either accepted or rejected out of network. On the other side target hospitals serve both their own (new) arrivals (class-1) and overflowed arrivals (class-2) from small-sized hospitals. Furthermore, patients of $H 1, H 2$ overflow to each other whenever one of them is full. The overflow stream of $H 1(H 2)$ is either accepted in hospital $H 2(H 1)$ or rejected out from network. An optimal admission control policy is required and explored for each service unit of $H 1$ and $H 2$.

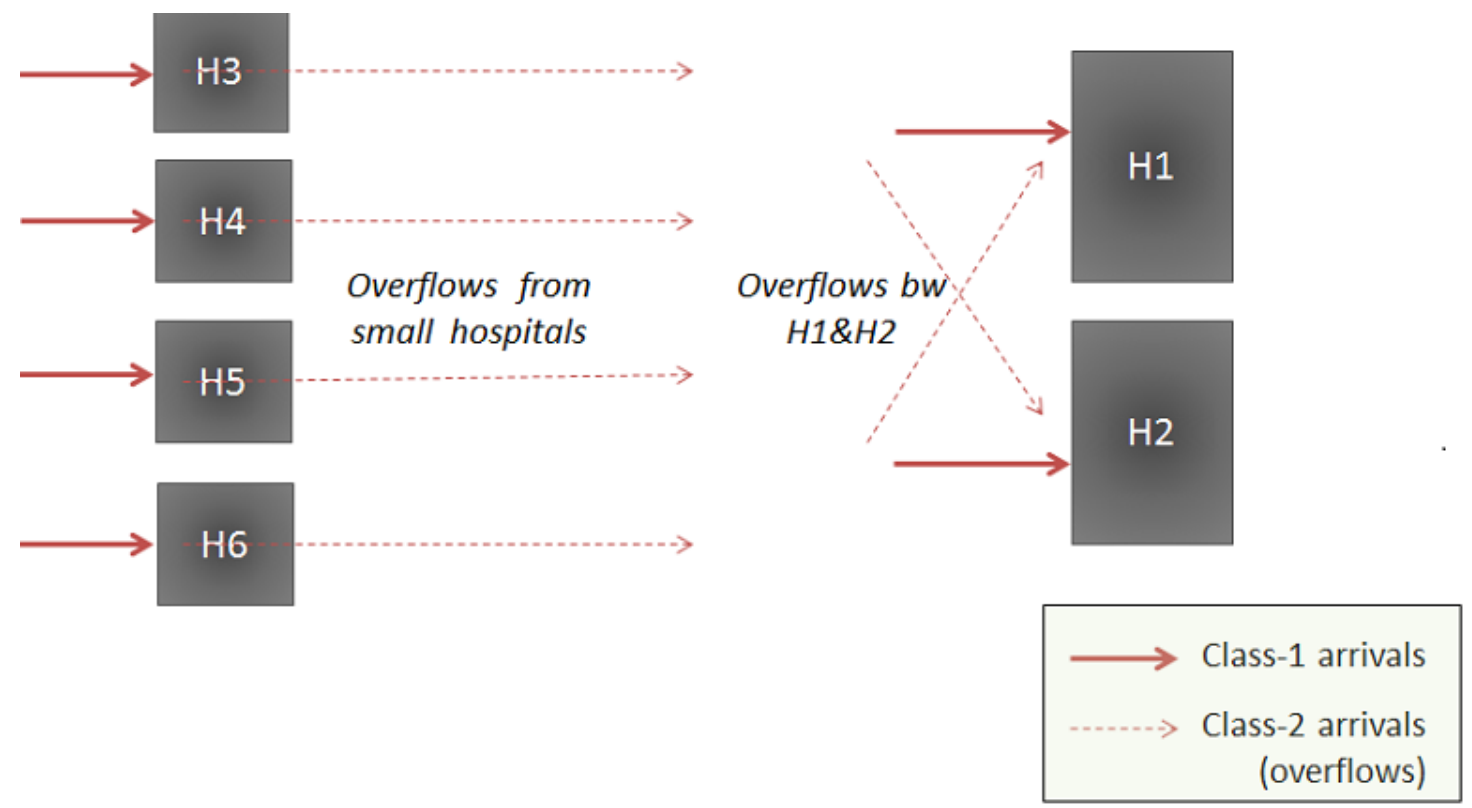

Figure 1: Perinatal Network Representation.

\subsection{Parameter Setting of Case Study}

This case study is partially supported by Agence Régionale de Santé (ARS), the regional health authority, provided us certain information and data. Unfortunately, data required for a simulation study is tremendous due to the large-scale of the work, therefore we construct the primary simulation model with adequate detail level. Poisson arrivals are often observed in systems without appointments but with arrivals controlled by nature such as birth delivery(Brandeau, Sainfort, and Pierskalla 2004). Therefore, class- 1 patients are assumed to arrive a service unit $s$ in hospital $i$ with a distribution of $\operatorname{Poisson}\left(\lambda_{s i}\right)$. For all obstetrics(OB) units in each hospital, ARS provides us yearly arrival data of each pregnant woman, their domicile and preferred hospital information. By assuming no seasonality within the year, we were able to compute daily offered load of each hospital in the network. ARS also provided the ratio of neonates requiring basic and intensive care, from where the arrival rate of neonates requiring basic and intensive care are computed. Furthermore, in heavily stochastic systems, the coefficient of variation (CV) of length of stay (LOS), which 
is defined as the ratio of the standard deviation to the mean, is typically close to one, satisfying the usage of negative exponential distribution as a service time assumption.

Servers are staffed-beds which is the combination of physical equipment (bed) with an appropriate coverage of nurses and physicians (De Bruin et al. 2010). In our applications, typical ratios are 5 beds per nurse in an obstetric unit and 2 beds per nurse in NICU. Each service unit $s$ in each hospital $i$ has multiple identical beds as servers $\left(N_{s i}\right)$ and each patient requires one bed with an i.i.d exponential service time $\left(\mu_{s i}\right)$ (same for both classes of patients).

Arrival rates and existing bed capacity of each service unit in each maternity facility is given in Table 1 from where the information about the type of hospitals can be extracted.

Table 1: Arrival rates $\left(\lambda_{s i}\right)$ and Existing Capacity $\left(N_{s i}\right)$

\begin{tabular}{c|cc|cc|cc|} 
& \multicolumn{2}{|c|}{ OB } & \multicolumn{2}{|c|}{ NEO } & \multicolumn{2}{c|}{ NICU } \\
\cline { 2 - 7 } & $\begin{array}{c}\text { New Arrivals } \\
\left(\lambda_{1 i}\right)\end{array}$ & $\begin{array}{c}\text { Bed Capacity } \\
\left(N_{1 i}\right)\end{array}$ & $\begin{array}{c}\text { New Arrivals } \\
\left(\lambda_{2 i}\right)\end{array}$ & $\begin{array}{c}\text { Bed Capacity } \\
\left(N_{2 i}\right)\end{array}$ & $\begin{array}{c}\text { New Arrivals } \\
\left(\lambda_{3 i}\right)\end{array}$ & $\begin{array}{c}\text { Bed Capacity } \\
\left(N_{3 i}\right)\end{array}$ \\
\hline H1 & 55 & 60 & 24 & 30 & 11 & 8 \\
H2 & 48 & 50 & 12 & 20 & 7 & 5 \\
H3 & 32 & 30 & 15 & 13 & - & - \\
H4 & 30 & 28 & 12 & 10 & - & - \\
H5 & 35 & 30 & - & - & - & - \\
H6 & 27 & 25 & - & - & - & - \\
\hline
\end{tabular}

\section{MARKOV DECISION PROCESS (MDP) MODEL}

In this section we formulate a general MDP model for admission control of patients in any of the service networks. In order to model the overflow system as an MDP process, we assume a Markovian system.

\subsection{Modeling Overflows}

Each small-sized hospital can be modeled as an M/M/N/N queue with Poisson arrivals, exponential service time distribution, $N$ identical beds and no waiting room. Then, rejection probability is given by the following Erlang loss function: $B_{s i}=\frac{\lambda \mu^{N} / N !}{\sum_{k=0}^{N} \lambda \mu^{k} / k !}$. Under this assumption, overflow rate from service unit $s$ of a small hospital $i$ is given as $\vartheta_{s i}=B_{s i} \lambda_{s i}, \forall s=\{1,2\}, i=\{3,4,5,6\}$ and they are assumed to be Poisson. Therefore, total overflow rate from small hospitals for service $s$ is $\vartheta_{s}=\sum_{i=3}^{6} \vartheta_{s i}$ and is also Poisson.

Remark 1: It is commonly known that overflow arrivals do not follow a Poisson distribution. This is a big but a necessary assumption in order to be able to use MDP since Poisson arrivals is an essential requirement in the application of MDP methods. This approximation signifies the importance of Simulation model to assess and compare the performance of MDP policy with other policies, consequently evaluate the impact of Markovian assumption on resulting output. Markovian approximation and its impact on admission probabilities and total reward is investigated with SIM.

Remark 2: As mentioned before, no patient overflow is assumed to occur among different service units. This fact let us to consider the three service networks independently(OBs, Neonatals, NICUs). Therefore, a general MDP model is formulated in the following section which can be used to describe the flow in each service network $s$.

\subsection{MDP Formulation}

Admission of patients to target hospitals $(H 1, H 2)$ is highly dependent on the number of occupied beds in both hospitals. Therefore, this system can be modeled as a two dimensional continuous time Markov 
Chain model with state $X=\left(x_{1}, x_{2}\right) \in \Omega$ defined as the number of occupied beds (number of patients) in $H 1$ and $H 2$ respectively where $\Omega=\left\{x_{1}, x_{2}: 0 \leqslant x_{1} \leqslant N_{1}\right.$ and $\left.0 \leqslant x_{2} \leqslant N_{2}\right\}$ is the state space.

A control action space is defined by a $2 X 2$ dimensional mapping matrix $A(X)=\left[a_{k i}(X)\right]$ with $a_{k i}(X)=$ $1(0)$ indicates the control action is to admit (reject) patient class $k=\{1,2\}$ at hospital $i=\{1,2\}$ when the system is in state $X$. There is a reward of $R_{k}$ associated with accepting a class- $k$ patient where $R_{1}>R_{2}$ so that, admission of a new patient is rewarded more than the overflow patient. Any class-k patient rejected from network brings no reward to the system. Some insights:

- $\quad a_{k i}(X)=0, \forall k, \forall i$ at state $X=\left(x_{1}=N_{1}, x_{2}=N_{2}\right)$ : When both hospitals are full, all arrivals are rejected.

- $a_{1 i}(X)=1, \forall i$ at states $x_{i}<N_{i}$ : Class- 1 patients are always admitted when the corresponding hospital is not full.

Our objective is to find an optimal control policy $\pi^{*}$ that maximizes the expected total discounted reward over an infinite horizon. Uniformization results in an equivalent discrete time Markov chain by allowing fictitious transitions from a state to itself. Using uniformization (Ross 1989), we express the DP optimality equation below. Therefore, Optimal Value Function for a service network $s$ can be written as:

$$
T V(X)=\alpha \sum_{1=1}^{2}\left(\frac{\alpha_{i}}{\gamma} T_{A_{i}} V(X)+\frac{\vartheta}{\gamma} T_{O A_{i}} V(X)+\frac{N_{i} \mu_{i}}{\gamma} T_{D_{i}} V(X)\right)
$$

where,

$\gamma=\sum_{i=1}^{2}\left(\lambda_{i}+N_{i} \mu_{i}\right)+\vartheta$ is uniformization rate, $\alpha$ is discount factor where $0<\alpha<1$,

The Arrival operator $T_{A_{i}}$ models admission control of class- 1 patients to hospital $i, j=\{1,2\}$, where $i \neq j$,

$T_{A_{i}} V(X)=\left\{\begin{array}{lr}r_{1}+V\left(X+e_{i}\right) & \text { for } x_{i}<N_{i} \\ \max \left\{r_{2}+V\left(X+e_{j}\right), V(X)\right\} & \text { for } x_{i}=N_{i} \text { and } x_{j}<N_{j} \\ V(X) & \text { for } X=N\end{array}\right.$

The Arrival operator $T_{O A_{i}}$ models admission control of overflow patients from small hospitals to hospital $i, j$

$T_{O A_{i}} V(X)=\left\{\begin{array}{lr}\max \left\{r_{2}+V\left(X+e_{i}\right), r_{2}+V\left(X+e_{j}\right), V(X)\right\} & \text { for } x_{i}<N_{i} \\ \max \left\{r_{2}+V\left(X+e_{j}\right), V(X)\right\} & \text { for } x_{i}=N_{i} \text { and } x_{j}<N_{j} \\ V(X) & \text { for } X=N\end{array}\right.$

The Departure operator $T_{D_{i}}$ models departures from hospital $i=\{1,2\}$

$T_{D_{i}} V(X)=\frac{x_{i}}{N_{i}} V\left(X-e_{i}\right)+\frac{N_{i}-x_{i}}{N_{i}} V(X) \quad$ for all $X$

- $e_{i}$ is the $i^{t h}$ unity vector

- $r_{i}$ is the unif. reward parameter where $r_{i}=R_{i} /(\beta+\gamma)$

An optimal control policy should be chosen in each state to maximize the future expected discounted revenue as given by the optimality equation.

\subsection{Computation of an optimal policy with MDP}

An optimal policy can be obtained by solving the DP value function analytically however explicit solution of such a function is not trivial. We utilize an efficient computational algorithm, Structured Value Iteration(VI), to reach the optimal policy for our case study.

When both of the hospitals are not full, it is intuitive and also proved in similar studies in literature that optimal action for class-2 patients is admission to the hospital which has the minimum number of beds occupied (shortest queue) proportional to their class-1 patient arrival rates. Therefore, in our case study in 


\section{Pehlivan, Augusto, and Xie}

the states $X=\left(x_{1}<N_{1}, x_{2}<N_{2}\right)$ overflow patients will be accepted to hospital $i\left(a_{2 i}(X)=1 a_{2 j}(X)=0\right)$ if $\frac{N_{i}-x_{i}}{\lambda_{i}}<\frac{N_{j}-x_{j}}{\lambda_{j}} \forall i, j=\{1,2\}$.

Therefore, optimal admission policy for certain states $X=\left(x_{1}<N_{1}, x_{2}<N_{2}\right)$ is already determined. In MDP model, we are interested in finding optimal actions for the states $x_{i}=N_{i}$ and $x_{j}<N_{j}$ (one hospital is full while other is not). With other words, we seek optimal threshold points at $H 1$ and $H 2$ above which overflowed arrivals will be rejected.

In the computational study, rewards for different class of patients are defined as $R 1=10, R 2=5$ and the discount factor $(\alpha)$ is taken very close to 1 . The proposed VI algorithm is given below:

Require: $\pi^{H 1}$ : the optimal policy for $H 1$ when $H 2$ is full $\pi^{H 2}$ : the optimal policy for $H 2$ when $H 1$ is full

Input: $\lambda, N($ given in Table 1$), \mu=1, R 1=10, R 2=5, \alpha=0.97$

\section{Initialize:}

st $\forall X \in \Omega: 0 \leqslant V_{0}(X) \leqslant V(X)$

$t:=0$

$\forall X, \pi_{0}^{H 1}(X)=1, \pi_{0}^{H 2}(X)=1, V_{0}(X)=0$

2. $t:=t+1$

\section{for all $X \in \Omega$ do}

$$
\begin{aligned}
& V_{t}(X)=\max _{a \in A(X)}\left\{\sum_{i=1}^{2} \alpha\left(\frac{\lambda_{i}}{\gamma} T_{A_{i}} V_{t-1}(X)+\frac{\vartheta}{\gamma} T_{O A_{i}} V_{t-1}(X)+\frac{N_{i} \mu_{i}}{\gamma} T_{D_{i}} V t-1(X)\right)\right\} \\
& \pi_{t}^{H 1}\left(x_{1}, N 2\right)=\arg \max _{a \in A\left(x_{1}, N 2\right)}\left\{\sum_{i=1}^{2} \alpha\left(\frac{\lambda_{i}}{\gamma} T_{A_{i}} V_{t-1}\left(x_{1}, N 2\right)+\frac{\vartheta}{\gamma} T_{O A_{i}} V_{t-1}\left(x_{1}, N 2\right)+\frac{N_{i} \mu_{i}}{\gamma} T_{D_{i}} V t-1\left(x_{1}, N 2\right)\right)\right\} \\
& \pi_{t}^{H 2}\left(N 1, x_{2}\right)=\arg \max _{a \in A\left(N 1, x_{2}\right)}\left\{\sum_{i=1}^{2} \alpha\left(\frac{\lambda_{i}}{\gamma} T_{A_{i}} V_{t-1}\left(N 1, x_{2}\right)+\frac{\vartheta}{\gamma} T_{O A_{i}} V_{t-1}\left(N 1, x_{2}\right)+\frac{N_{i} \mu_{i}}{\gamma} T_{D_{i}} V t-1\left(N 1, x_{2}\right)\right)\right\}
\end{aligned}
$$

\section{end for}

3. Compute:

$\operatorname{dif}_{\text {min }}=\min _{l \in \Omega}\left\{V_{t}(l)-V_{t-1}(l)\right\}$

$d i f_{\text {max }}=\max _{l \in \Omega}\left\{V_{t}(l)-V_{t-1}(l)\right\}$

if $d i f_{\text {max }}-d i f_{\text {min }} \leqslant \varepsilon \times d i f_{\text {min }}$ then

Policy $\pi_{t}^{H 1}\left(x_{1}, N 2\right)$ and $\pi_{t}^{H 2}\left(N 1, x_{2}\right)$ are optimal for $\mathrm{H} 1$ and $\mathrm{H} 2$ respectively

\section{Stop}

else

\section{Go to step 2.}

end if

The stationary policy defined in the value iteration algorithm converges to a $2 \mathrm{D}$ threshold based policy which proposes control points $x_{1}^{*}$ and $x_{2}^{*}$ such that:

- $\quad$ accept class-2 patients to $H 1$ while $\left(x_{1}, N 2\right)<\left(x_{1}^{*}, N 2\right)$ and reject while $\left(x_{1}, N 2\right) \geqslant\left(x_{1}^{*}, N 2\right)$

- $\quad$ accept class-2 patients to $H 2$ while $\left(N 1, x_{2}\right)<\left(N 1, x_{2}^{*}\right)$ and reject while $\left(N 1, x_{2}\right) \geqslant\left(N 1, x_{2}^{*}\right)$

The value iteration algorithm is solved independently for three service networks and the optimal policies are found as:

Optimal Policy for Obstetrics $(s=1): \quad x_{1}^{*}=58$ and $x_{2}^{*}=48$

Optimal Policy for Neonatals $(s=2): \quad x_{1}^{*}=29$ and $x_{2}^{*}=19$

Optimal Policy for NICUs (s=3): $\quad x_{1}^{*}=7$ and $x_{2}^{*}=4$ 


\section{DISCRETE EVENT SIMULATION}

In this section we build our simulation model which serves as a practical platform to generate and evaluate various policy scenarios and strengthens the decision making process by incorporating the complexity in the model which can not be captured by MDP. With the results of those scenarios, Sim model will be able to measure the impact of crucial Markovian assumption posed in MDP model (Poisson distributed overflow streams), the impact of different control policies on admission probabilities and corresponding expected total rewards.

The Sim model is constructed in a commercial software Rockwell Arena 2011. Results are tested in a computer $2.67 \mathrm{GHz}$ and $6 \mathrm{Go}$.

As simulation output, the relevant performance indicators are taken as the admission probabilities of each arriving stream to target hospitals. From admission probabilities, the total reward is calculated by using the unit rewards per patient $R 1$ and $R 2$. Admission probabilities are defined in table 2 .

Table 2: Simulation Output

\begin{tabular}{l|l}
$p_{i}^{s}$ & Probability of class- 1 patients admitted in service unit $s$ in hospital $i=\{1,2\}$ \\
\hline$p_{j i}^{s}$ & $\begin{array}{l}\text { Probability of } \text { class }-2 \text { patients rejected from service unit } s \text { of hospital } j= \\
\{1,2,3,4,5,6\} \text { and admitted in service unit } s \text { of hospital } i=\{1,2\}\end{array}$ \\
\hline
\end{tabular}

Total Reward for each service network $s$ :

$$
\text { Total Reward }=R 1 \cdot\left(\sum_{i=1}^{2} \lambda_{s i} p_{i}^{s}\right)+R 2 \cdot\left(\sum_{j=1}^{6} \lambda_{s j}\left(\sum_{i=1}^{2} p_{j i}^{s}\right)\right)
$$

where $p_{j i}^{s}=0$ for $i=j$

In order to simulate the actual system accurately, some important simulation parameters are to be determined such as number of replications, replication length and warm up period. In order to ensure a $95 \%$ confidence interval for performance indicators, 20 replications are launched. The minimal amount of replications $n$ is calculated using the following formula (Law and Kelton 1984): $\bar{X}(n) \pm t_{n-1,1-\alpha / 2} \sqrt{\frac{S^{2}(n)}{n}}$. $S^{2}(n)$ is the estimated standard deviation and $\bar{X}(n)$ is the estimated average for the $X_{1}, X_{2}, \ldots, X_{n}$ values of an indicator for $n$ replications. The value $t_{n-1,1-\alpha / 2}$ is the critical point of the distribution $t$ with $n-1$ degrees of liberty and a covering equal to $\frac{1-\alpha}{2}$ where $1-\alpha=0.95$.

In order to determine run length and warm up period, we have observed the bed occupancies in the system. Since our model is a pure loss system, there is no queue, therefore entities in the system do not grow exponentially in time and simulation model can reach its steady state condition quickly. For each control policy scenario, SIM model is run with 20 replications, with a run length of 10000 days and with a warm-up period of 100 days. We were able to verify the simulation model by counting on different methods. Firstly, we monitored the flow of entities and confirmed the conformed behavior of the model to the reality. Secondly, we were able to use Markov Chain modeling for a specific scenario in order to verify the SIM model. The system under no control policy (all arrivals are accepted unless hospitals are full) has fewer variables to deal with, therefore we were able to model this system as a Markov Chain. For that specific case, the exact results of Markov model were very close to the results obtained from SIM model, set with the parameters above.

\subsection{Computational Results}

In this section, we present the SIM model results and comparison with the MDP optimal policies obtained for obstetric $(s=1)$ and basic neonatal $(s=2)$ services. NICUs are not considered in the simulation runs. 
In the network only the target hospitals $(H 1$ and $H 2)$ contain NICU units; no external overflow streams (from small hospitals) occurs, so that no assumption of poisson is needed to be considered. Therefore, policy proposed by MDP model is optimal.

The optimal policies obtained from MDP model for obstetrics and neonatal services are used as base scenarios for SIM model. MDP model gives an optimal policy of $x_{1}^{*}=58$ and $x_{2}^{*}=48$ (base scenario-OB) and $x_{1}^{*}=29$ and $x_{2}^{*}=19$ (base scenario-Neo) respectively for Obstetrics and Neonatal Units in $H 1$ and H2. Several control policy scenarios are generated around these base scenarios to be tested in SIM model. Scenarios are determined by modifying the control parameters $x_{1}^{*}$ and $x_{2}^{*}$ of base scenario-OB and base scenario-Neo.

Resulting admission probabilities of each scenario and their 95\% confidence intervals are given in Table 3 and Table 4 respectively for Obstetric and Neonatal units. (All of the policies tested could not be presented here due to page restrictions). As $x_{1}^{*}$ and $x_{2}^{*}$ get smaller, it is expected that admission probabilities of class- 1 patients $p_{i}^{s}$ increase while admission probabilities of class-2 patients $p_{j i}^{s}$ decrease since more beds are reserved for class- 1 patients.

Table 3: Admission Probabilities computed for various scenarios for Obstetrics Units

\begin{tabular}{|c|c|c|c|c|c|c|c|c|c|c|c|c|c|c|}
\hline$x_{1}{ }^{*}$ & $x_{2}{ }^{*}$ & $p_{1}$ & $p_{2}$ & $p_{12}$ & $p_{21}$ & $p_{31}$ & $p_{32}$ & $p_{41}$ & $p_{42}$ & $p_{51}$ & $p_{52}$ & $p_{61}$ & $p_{62}$ & $\begin{array}{l}\text { Total } \\
\text { Rew. }\end{array}$ \\
\hline 60 & 50 & $\begin{array}{c}0.674 \\
\pm \\
0.02 \\
\end{array}$ & $\begin{array}{c}0.61 \\
\pm \\
0.018\end{array}$ & $\begin{array}{c}0.153 \\
\pm \\
0.02\end{array}$ & $\begin{array}{c}0.104 \\
\pm \\
0.013 \\
\end{array}$ & $\begin{array}{c}0.099 \\
\pm \\
0.002\end{array}$ & $\begin{array}{c}0.098 \\
\pm \\
0.0017\end{array}$ & $\begin{array}{c}0.135 \\
\pm \\
0.003\end{array}$ & $\begin{array}{c}0.133 \\
\pm \\
0.002\end{array}$ & $\begin{array}{c}0.109 \\
\pm \\
0.0024\end{array}$ & $\begin{array}{c}0.106 \\
\pm \\
0.0011 \\
\end{array}$ & $\begin{array}{c}0.094 \\
\pm \\
0.002\end{array}$ & $\begin{array}{c}0.092 \\
\pm \\
0.0014\end{array}$ & 807.1 \\
\hline 59 & 49 & $\begin{array}{c}0.766 \\
\pm \\
0.008\end{array}$ & $\begin{array}{c}0.688 \\
\pm \\
0.008\end{array}$ & $\begin{array}{c}0.082 \\
\pm \\
0.01\end{array}$ & $\begin{array}{c}0.049 \\
\pm \\
0.006\end{array}$ & $\begin{array}{c}0.086 \\
\pm \\
0.0003\end{array}$ & $\begin{array}{c}0.092 \\
\pm \\
0.0008\end{array}$ & $\begin{array}{c}0.11 \\
\pm \\
0.0006\end{array}$ & $\begin{array}{c}0.12 \\
\pm \\
0.0008\end{array}$ & $\begin{array}{c}0.093 \\
\pm \\
0.0005\end{array}$ & $\begin{array}{c}0.1 \\
\pm \\
0.0008\end{array}$ & $\begin{array}{c}0.081 \\
\pm \\
0.0003\end{array}$ & $\begin{array}{c}0.087 \\
\pm \\
0.0007\end{array}$ & 845.6 \\
\hline 58 & 48 & $\begin{array}{c}0.796 \\
\pm \\
0.004\end{array}$ & $\begin{array}{c}0.715 \\
\pm \\
0.0044 \\
\end{array}$ & $\begin{array}{c}0.05 \\
\pm \\
0.006\end{array}$ & $\begin{array}{c}0.027 \\
\pm \\
0.003\end{array}$ & $\begin{array}{c}0.083 \\
\pm \\
0.0\end{array}$ & $\begin{array}{c}0.09 \\
\pm \\
0.0006\end{array}$ & $\begin{array}{c}0.11 \\
\pm \\
0.0002\end{array}$ & $\begin{array}{c}0.122 \\
\pm \\
0.0008\end{array}$ & $\begin{array}{c}0.091 \\
\pm \\
0.0001\end{array}$ & $\begin{array}{c}0.098 \\
\pm \\
0.0004\end{array}$ & $\begin{array}{c}0.078 \\
\pm \\
0.0001\end{array}$ & $\begin{array}{c}0.084 \\
\pm \\
0.0004\end{array}$ & 856.8 \\
\hline 56 & 47 & $\begin{array}{c}0.819 \\
\pm \\
0.0016\end{array}$ & $\begin{array}{c}0.734 \\
\pm \\
0.0021\end{array}$ & $\begin{array}{c}0.02 \\
\pm \\
0.003\end{array}$ & $\begin{array}{c}0.015 \\
\pm \\
0.002\end{array}$ & $\begin{array}{c}0.082 \\
\pm \\
0.0\end{array}$ & $\begin{array}{c}0.087 \\
\pm \\
0.0003\end{array}$ & $\begin{array}{c}0.112 \\
\pm \\
0.00\end{array}$ & $\begin{array}{c}0.119 \\
\pm \\
0.0002\end{array}$ & $\begin{array}{c}0.09 \\
\pm \\
0.0\end{array}$ & $\begin{array}{c}0.095 \\
\pm \\
0.0005\end{array}$ & $\begin{array}{c}0.077 \\
\pm \\
0.0002\end{array}$ & $\begin{array}{c}0.082 \\
\pm \\
0.0002\end{array}$ & 865.4 \\
\hline 56 & 44 & $\begin{array}{c}0.821 \\
\pm \\
0.0015\end{array}$ & $\begin{array}{c}0.743 \\
\pm \\
0.0008\end{array}$ & $\begin{array}{c}0.02 \\
\pm \\
0.0016\end{array}$ & $\begin{array}{c}0.003 \\
\pm \\
0.0004\end{array}$ & $\begin{array}{c}0.081 \\
\pm \\
0.0002\end{array}$ & $\begin{array}{c}0.087 \\
\pm \\
0.0004\end{array}$ & $\begin{array}{c}0.111 \\
\pm \\
0.0\end{array}$ & $\begin{array}{c}0.12 \\
\pm \\
0.0003\end{array}$ & $\begin{array}{c}0.089 \\
\pm \\
0.0\end{array}$ & $\begin{array}{c}0.096 \\
\pm \\
0.0004\end{array}$ & $\begin{array}{c}0.077 \\
\pm \\
0.0002\end{array}$ & $\begin{array}{c}0.082 \\
\pm \\
0.0\end{array}$ & 867.1 \\
\hline 55 & 43 & $\begin{array}{c}0.826 \\
\pm \\
0.0009\end{array}$ & $\begin{array}{c}0.748 \\
\pm \\
0.0006\end{array}$ & $\begin{array}{c}0.01 \\
\pm \\
0.0017\end{array}$ & $\begin{array}{c}0.002 \\
\pm \\
0.0002\end{array}$ & $\begin{array}{c}0.082 \\
\pm \\
0.0001\end{array}$ & $\begin{array}{c}0.086 \\
\pm \\
0.0002\end{array}$ & $\begin{array}{c}0.112 \\
\pm \\
0.0001\end{array}$ & $\begin{array}{c}0.119 \\
\pm \\
0.0001\end{array}$ & $\begin{array}{c}0.09 \\
\pm \\
0.0\end{array}$ & $\begin{array}{c}0.095 \\
\pm \\
0.0004\end{array}$ & $\begin{array}{c}0.077 \\
\pm \\
0.0002\end{array}$ & $\begin{array}{c}0.081 \\
\pm \\
0.0001\end{array}$ & 870 \\
\hline 55 & 42 & $\begin{array}{c}0.825 \\
\pm \\
0.0009 \\
\end{array}$ & $\begin{array}{c}0.748 \\
\pm \\
0.0004 \\
\end{array}$ & $\begin{array}{c}0.013 \\
\pm \\
0.0017\end{array}$ & $\begin{array}{c}0.001 \\
\pm \\
0.0001 \\
\end{array}$ & $\begin{array}{c}0.081 \\
\pm \\
0.0002\end{array}$ & $\begin{array}{c}0.087 \\
\pm \\
0.0003\end{array}$ & $\begin{array}{c}0.112 \\
\pm \\
0.0\end{array}$ & $\begin{array}{c}0.119 \\
\pm \\
0.0 \\
\end{array}$ & $\begin{array}{c}0.09 \\
\pm \\
0.0 \\
\end{array}$ & $\begin{array}{c}0.095 \\
\pm \\
0.0004\end{array}$ & $\begin{array}{c}0.077 \\
\pm \\
0.0002 \\
\end{array}$ & $\begin{array}{c}0.082 \\
\pm \\
0.0001\end{array}$ & 869 \\
\hline 55 & 41 & $\begin{array}{c}0.825 \\
\pm \\
0.0009 \\
\end{array}$ & $\begin{array}{c}0.746 \\
\pm \\
0.0006\end{array}$ & $\begin{array}{c}0.014 \\
\pm \\
0.0017 \\
\end{array}$ & $\begin{array}{c}0.001 \\
\pm \\
0.0\end{array}$ & $\begin{array}{c}0.082 \\
\pm \\
0.0 \\
\end{array}$ & $\begin{array}{c}0.087 \\
\pm \\
0.0002\end{array}$ & $\begin{array}{c}0.112 \\
\pm \\
0.0\end{array}$ & $\begin{array}{c}0.118 \\
\pm \\
0.0\end{array}$ & $\begin{array}{c}0.09 \\
\pm \\
0.0\end{array}$ & $\begin{array}{c}0.095 \\
\pm \\
0.0004\end{array}$ & $\begin{array}{c}0.077 \\
\pm \\
0.0003\end{array}$ & $\begin{array}{c}0.082 \\
\pm \\
0.0001\end{array}$ & 868.4 \\
\hline 0 & 0 & $\begin{array}{c}0.831 \\
\pm \\
0.0002 \\
\end{array}$ & $\begin{array}{c}0.751 \\
\pm \\
0.0001\end{array}$ & 0 & 0 & $\begin{array}{c}0.082 \\
\pm \\
0.0001\end{array}$ & $\begin{array}{c}0.085 \\
\pm \\
0.0003\end{array}$ & $\begin{array}{c}0.112 \\
\pm \\
0.0\end{array}$ & $\begin{array}{c}0.117 \\
\pm \\
0.0\end{array}$ & $\begin{array}{c}0.09 \\
\pm \\
0.0\end{array}$ & $\begin{array}{c}0.094 \\
\pm \\
0.0002\end{array}$ & $\begin{array}{c}0.077 \\
\pm \\
0.0003\end{array}$ & $\begin{array}{c}0.081 \\
\pm \\
0.0001\end{array}$ & 869.7 \\
\hline
\end{tabular}

Figure 2 presents Total Rewards calculated for each control scenario simulated for Obstetrics service network. The maximum total reward is 870 and it is achieved at the control points $x_{1}^{*}=55$ and $x_{2}^{*}=43$. SIM optimal policy provides $7 \%$ increase in the total reward compared to no control policy case. The optimal control points proposed by SIM model is different than the ones obtained from MDP model as expected due to Markovian assumption. However, it is important to note that \% difference between total rewards obtained from both policies is not significant such that total best reward of SIM is $1.5 \%$ higher than the one of MDP. Futhermore, it can be clearly observed from Figure 2 that reserving two beds in each hospital (as proposed by MDP) brings the biggest rise in total reward. Reserving more beds until 
Table 4: Admission Probabilities computed for various scenarios for Neonatal Units

\begin{tabular}{|l|c|c|c|c|c|c|c|c|c|c|}
\hline $\boldsymbol{x}_{\mathbf{1}}{ }^{*}$ & $\boldsymbol{x}_{\mathbf{2}}{ }^{*}$ & $\boldsymbol{p}_{\mathbf{1}}$ & $\boldsymbol{p}_{\mathbf{2}}$ & $\boldsymbol{p}_{\mathbf{1 2}}$ & $\boldsymbol{p}_{\mathbf{2 1}}$ & $\boldsymbol{p}_{\mathbf{3 1}}$ & $\boldsymbol{p}_{\mathbf{3 2}}$ & $\boldsymbol{p}_{\mathbf{4 1}}$ & $\boldsymbol{p}_{\mathbf{4 2}}$ & $\begin{array}{l}\text { Total } \\
\text { Reward }\end{array}$ \\
\hline $\mathbf{3 0}$ & $\mathbf{2 0}$ & 0.862 & 0.845 & 0.091 & 0.074 & 0.135 & 0.141 & 0.183 & 0.193 & $\mathbf{3 6 7 . 3 8}$ \\
& & \pm & \pm & \pm & \pm & \pm & \pm & \pm & \pm & \\
& & 0.0006 & 0.0005 & 0.0003 & 0.0002 & 0.0001 & 0.0003 & 0.0003 & 0.0003 & \\
\hline $\mathbf{2 9}$ & $\mathbf{1 9}$ & 0.885 & 0.88 & 0.06 & 0.053 & 0.127 & 0.14 & 0.172 & 0.192 & $\mathbf{3 7 1 . 2 8}$ \\
& & \pm & \pm & \pm & \pm & \pm & \pm & \pm & \pm & \\
& & 0.002 & 0.004 & 0.0024 & 0.004 & 0.0011 & 0.0003 & 0.0012 & 0.0001 & \\
\hline $\mathbf{2 8}$ & $\mathbf{1 8}$ & 0.894 & 0.894 & 0.044 & 0.042 & 0.123 & 0.139 & 0.168 & 0.19 & $\mathbf{3 7 2 . 0 9}$ \\
& & \pm & \pm & \pm & \pm & \pm & \pm & \pm & \pm & \\
& & 0.003 & 0.005 & 0.003 & 0.006 & 0.0016 & 0.0002 & 0.002 & 0.0002 & \\
\hline $\mathbf{2 7}$ & $\mathbf{1 8}$ & 0.897 & 0.896 & 0.036 & 0.041 & 0.122 & 0.139 & 0.166 & 0.19 & $\mathbf{3 7 2 . 2 4}$ \\
& & \pm & \pm & \pm & \pm & \pm & \pm & \pm & \pm & \\
& & 0.003 & 0.005 & 0.004 & 0.007 & 0.0016 & 0.0002 & 0.0021 & 0.0003 & \\
\hline $\mathbf{2 5}$ & $\mathbf{1 8}$ & 0.901 & 0.899 & 0.025 & 0.04 & 0.121 & 0.137 & 0.166 & 0.188 & $\mathbf{3 7 2 . 4 5}$ \\
& & \pm & \pm & \pm & \pm & \pm & \pm & \pm & \pm & \\
& & 0.004 & 0.005 & 0.004 & 0.008 & 0.0018 & 0.0003 & 0.0022 & 0.0005 & \\
\hline
\end{tabular}

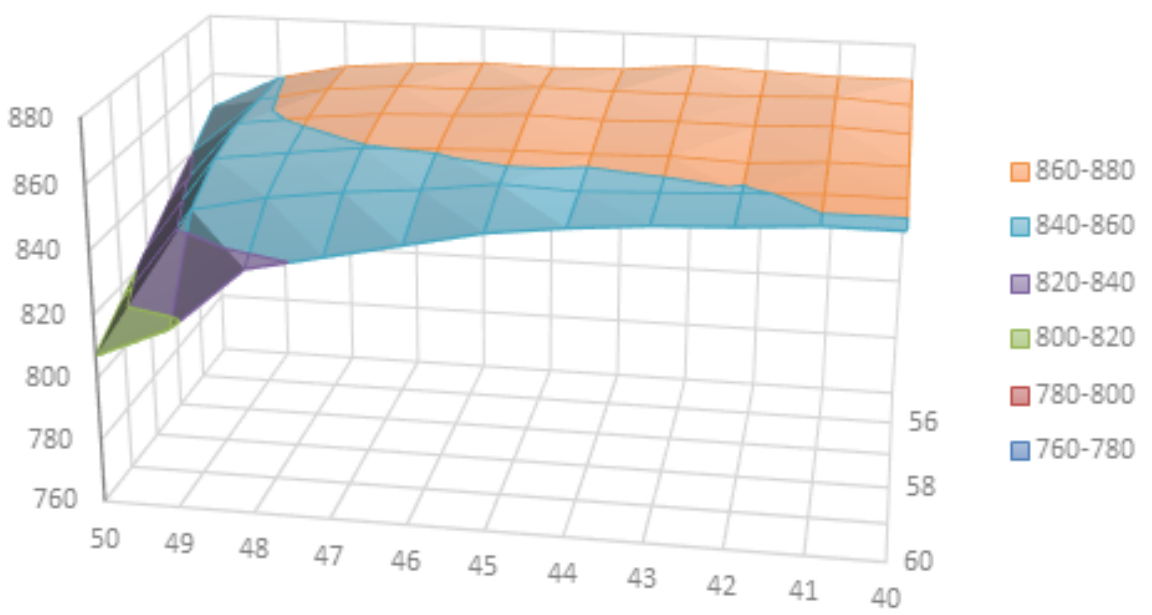

Figure 2: Total Rewards obtained for each scenario in Obstetric Units

reaching the SIM optimal points helps to increase total reward to its max level but with a declined slope. Additionally, reserving more beds after reaching the optimal points proposed by SIM does not have any significant effect on total reward.

Figure 3 presents Total Rewards calculated for each control scenario simulated for basic neonatal service network. The maximum total reward is 372.5 and it is achieved at the control points $x_{1}^{*}=25$ and $x_{2}^{*}=18$, on the other hand total rewards obtained from the control scenarios lie in the blue region in Figure 3 are quite close to the attained maximum reward, therefore those policies can all be considered as alternative optimum policies. Again as expected, the optimal control policies proposed by SIM model are different than the MDP optimal control policy due to the Markovian assumption. However, \% difference between total rewards is not significant such that the max total reward obtained from SIM is $0.3 \%$ higher than the total reward of MDP optimal policy which is computed as 371.28.

In general it can be concluded for neonatal units that reserving beds (posing an admission control policy) do not make a big impact on admission probabilities (given in Table 4), consequently we do not 
observe a significant $\%$ improvement in total rewards. If we compare the total rewards obtained from the optimal policy of SIM and no control policy, we can state that \% difference does not exceed $1.5 \%$.

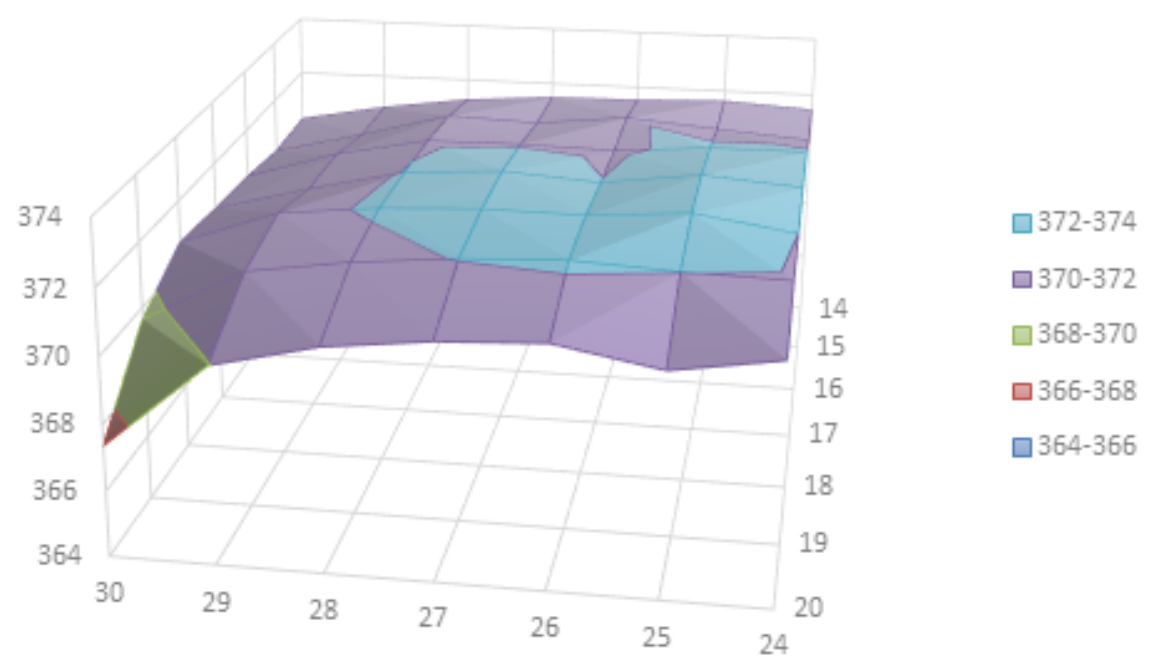

Figure 3: Total Rewards obtained for each scenario in Neonatal Units

\section{CONCLUSION AND FUTURE WORK}

In this paper, we considered the problem of finding an optimal admission control policy for two target hospitals in a pure-loss perinatal network. In order to model the system analytically, a Markovian assumption was necessary to make. Under this assumption, the system is modeled as an MDP process and an MDP optimal policy is computed. Afterwards, simulation model is used to strengthen the decision making process by incorporating the complexity in the model which can not be captured by MDP and measure the impact of Markovian assumptions on total reward. For that aim, MDP optimal policy is considered as a base scenario and various control scenarios are generated around the base scenarios and evaluated with the simulation model. It has been observed that Simulation model proposes to reserve more beds in both of the hospitals in its optimal scenarios compared to MDP optimal policies. Final total reward obtained from SIM optimal policies is higher than the total reward obtained from MDP optimal policies. However, the percentage differences are not always significant changing with the considered instance.

In this study, overflowed patients are assumed to arrive to two target hospitals and admission control policies are investigated only for those hospitals. Even though this assumption is realistic for our case study held in a specific perinatal network, in general in pure-loss networks such as "network of emergency units" overflow arrivals are not restricted with two target hospitals. For a future work, we believe it is scientifically interesting to consider a pure-loss healthcare network where overflows are less restricted as in reality and deal with the problem of finding optimal admission control policies for all hospitals in the network.

\section{REFERENCES}

Brailsford, S. C. 2007. "Advances and challenges in healthcare simulation modeling: tutorial". In Proceedings of the 2007 Winter Simulation Conference, edited by S. Henderson, B. Biller, M. Hsieh, J. Shortle, J. Tew, and R. Barton, 1436-1448. Piscataway, New Jersey: Institute of Electrical and Electronics Engineers (IEEE). 
Brandeau, M. L., F. Sainfort, and W. P. Pierskalla. 2004. Operations research and health care: A handbook of methods and applications, Volume 70. Springer.

Charfeddine, M., and B. Montreuil. 2010. "Integrated agent-oriented modeling and simulation of population and healthcare delivery network: application to COPD chronic disease in a Canadian region". In Proceedings of the 2010 Winter Simulation Conference, edited by B. Johansson, S. Jain, J. MontoyaTorres, J. Hugan, and E. Yucesan, 2327-2339. Piscataway, New Jersey: Institute of Electrical and Electronics Engineers (IEEE).

De Bruin, A., R. Bekker, L. Van Zanten, and G. Koole. 2010. "Dimensioning hospital wards using the Erlang loss model". Annals of Operations Research 178 (1): 23-43.

Delasay, M., B. Kolfal, and A. Ingolfsson. 2012. "Maximizing throughput in finite-source parallel queue systems". European Journal of Operational Research 217 (3): 554-559.

$\mathrm{Ku}, \mathrm{C} .-$ Y., and S. Jordan. 2002. "Access control of parallel multiserver loss queues". Performance Evaluation 50 (4): 219-231.

Law, A. M., and W. D. Kelton. 1984. "Confidence intervals for steady-state simulations: I. A survey of fixed sample size procedures". Operations Research 32 (6): 1221-1239.

Miller, M., D. Ferrin, and N. Shahi. 2009. "Estimating patient surge impact on boarding time in several regional emergency departments". In Proceedings of the 2009 Winter Simulation Conference, edited by M. Rossetti, R. Hill, B. Johansson, A. Dunkin, and R. Ingalls, 1906-1915. Piscataway, New Jersey: Institute of Electrical and Electronics Engineers (IEEE).

Pehlivan, C., V. Augusto, X. Xie, and C. Crenn-Hebert. 2012. "Multi-period capacity planning for maternity facilities in a perinatal network: A queuing and optimization approach". In Automation Science and Engineering (CASE), 2012 IEEE International Conference on, 137-142. IEEE.

Roberts, S. D. 2011. "Tutorial on the simulation of healthcare systems". In Proceedings of the 2011 Winter Simulation Conference, edited by S. Jain, R. R. Creasey, J. Himmelspach, K. P. White, and M. Fu, 1408-1419. Piscataway, New Jersey: Institute of Electrical and Electronics Engineers (IEEE).

Ross, K. W. 1989. "Randomized and pastdependent policies for Markov decision processes with multiple constraints". Operations Research 37 (3): 474-477.

\section{AUTHOR BIOGRAPHIES}

CANAN PEHLIVAN is a Ph.D. Student at Center for Health Engineering at Ecole Nationale Superieure des Mines de Saint Etienne, France. She received her MSc degree in Industrial Engineering from Middle East Technical University (METU), Turkey, in 2008. Her research interests include capacity and location planning, applications of simulation methods and Markov Theory in healthcare systems. Her email address is pehlivan@emse.fr

VINCENT AUGUSTO received the Ph.D. degree in industrial engineering from the Ecole Nationale Superieure des Mines de Saint-Etienne, France, in 2009. Currently, he is an Assistant Professor with the Department of Healthcare Engineering, ENSM.SE. He was a visiting scholar with CIRRELT, University of Laval, Quebec, QC, Canada, in 2009. His research interests include modeling, simulation, and optimization of healthcare systems and their supply chains. His email address is augusto@emse.fr.

XIAOLAN XIE received the Ph.D. degree from University of Nancy I, Nancy, France, in 1989, and the Habilitation á Diriger des Recherches degree from University of Metz, France, in 1995. Currently, he is a Professor of industrial engineering and Head of Healthcare Engineering Department of Center for Health Engineering, Ecole Nationale Superieure des Mines de Saint-Etienne (ENSM.SE), France. He is also a Chair Professor with Shanghai Jiao Tong University (SJTU), China. Before joining ENSM.SE, he was a Research Director with the Institut National de Recherche en Informatique et en Automatique (INRIA), Metz, France, from 2002 to 2005; a Full Professor with Ecole Nationale d'ingenieurs de Metz, France, from 1999 to 2002; and a Senior Research Scientist at INRIA from 1990 to 1999. He is author/coauthor of over 
200 publications including over 80 journal articles and five books. His research interests include design, planning and scheduling, supply chain optimization, performance evaluation, maintenance of manufacturing and healthcare systems. Prof. Xie has been an Associate Editor on the conference Editorial Board of IEEE Robotics and Automation Society, IEEE Transactions on Automation Science and Engineering, IEEE Transaction on Automatic Control, IEEE Transactions on Robotics and Automation. His email address is xie@emse.fr. 